\title{
Second Quantized Reduced Bloch Equations and the Exact Solutions for Pairing Hamiltonian
}

\author{
Liqiang Wei \\ Institute for Theoretical Atomic, Molecular and Optical Physics \\ Harvard University, Cambridge, MA 02138 \\ Chiachung Sun \\ Institute of Theoretical Chemistry, Jilin University \\ Changchun, Jilin 130023 P. R. China
}

October 29, 2018

\begin{abstract}
In this article, we present a set of hierarchy Bloch equations for the reduced density operators in either canonical or grand canonical ensembles in the occupation number representation. They provide a convenient tool for studying the equilibrium quantum statistical mechanics for some model systems. As an example of their applications, we solve the equations for the model system with a pairing Hamiltonian. With the aid of its symplectic group symmetry, we obtain the statistical reduced density matrices with different orders. As a special instance for the solutions, we also get the reduced density matrices of the ground state for a superconductor.
\end{abstract}




\section{Introduction}

The language of the second quantization is a convenient and ubiquitous tool for studying the quantum many-body systems in modern physics. The quantum statistics of the identical many particles is automatically satisfied by the commutation relations of the creation or annihilation operators, and the complicated mathematical manipulation with wavefunctions such as Slater determinants and with matrix elements of operators is significantly simplified. It is no surprise that a familiarity with the related knowledge of the occupation number representation is a must for a serious theorist.

In a recent paper, we have developed an equation of motion approach for a direct determination of the reduced density matrices for the statistical ensembles under the framework of equilibrium quantum statistical mechanics [1-4]. We have derived the hierarchy Bloch equations which provide the laws according to which the reduced statistical density operators vary in temperature. We also take the orbital approximation, and solve the equations for the identical fermion systems with two-body interaction in a grand canonical ensemble. We have gained significant new physical insights. We find that the usual Fermi-Dirac statistics for the free fermion gas holds also for the interacting fermions, and that the usual Hartree-Fock equation at zerotemperature can be extended to the case of any finite temperature. The mean force field that every orbital or particle is subject to will be from the contribution of both coherent and incoherent superpositions of the wavefunctions of other orbitals or particles, and is therefore temperature-dependent [5-8]. This opens a way for investigating the interplay between the microscopic structure and macroscopic thermodynamic quantities for molecules 
and solids [5-9]. In addition, the Fermi-Dirac distribution for interacting particles demonstrates that there exists an effective one-particle description for the interacting many-body system $[2,10]$.

In the current paper, we begin the presentation of the scheme for an exact solution of the reduced Bloch equations which will be developed in a second quantized form. In quantum many-body theory, the systems are often represented by the model Hamiltonians in an occupation number representation. We study the pairing Hamiltonian, which is a useful model for the study of nuclear structure $[11,12]$, microscopic state of superconductors [13,14], and electronic structure of molecules $[15,16]$.

We organize this paper as follows. In the next Section, starting from the Bloch equation, we deduce a set of hierarchy equations in the occupation number representation for the reduced density operators in the canonical ensemble. The derivation is based on the second quantized form of the contraction operator which is presented in the Appendix. We also extend it to the case of a grand canonical ensemble. In Section 3, we first briefly review the symplectic group symmetry of the pairing Hamiltonian and present the second quantized form of its eigenvectors characterized by the particle number, the weight and the seniority number. Then using the symmetric conditions among $D^{1}, D^{2}$ and $D^{3}$, we solve the reduced Bloch equations in the canonical ensemble for the pairing Hamiltonian and obtain its canonical reduced density matrices with different orders. As a special situation, they yield the reduced density matrices of the ground state for the superconductor. We conclude our discussion in the final Section. 


\section{Reduced Bloch Equations in the Occupa- tion Number Representation}

We consider an identical $N$-fermion system with a two-body interaction. Its Hamiltonian has the following form,

$$
H=\sum_{i=1}^{N} h(i)+\sum_{i<j}^{N} v(i, j),
$$

where $h(i)$ and $v(i, j)$ are one- and two-particle operators, respectively. Assume a generic and complete set of single-particle states $\{\mid i>\}$, which form a single-particle Hilbert space $V$. For each $|i\rangle$, the corresponding creation or annihilation operators are defined as

$$
\left|i>=a_{i}^{+}\right| 0>, a_{i}\left|j>=\delta_{i j}\right| 0>\text {. }
$$

They satisfy the anticommutation relations,

$$
\begin{aligned}
\left\{a_{i}, a_{j}^{+}\right\} & =\delta_{i j}, \\
\left\{a_{i}, a_{j}\right\}=\left\{a_{i}^{+}, a_{j}^{+}\right\} & =0 .
\end{aligned}
$$

Define an $N$-fold antisymmetrized direct product space of the single-particle Hilbert space $V$ as

$$
V^{N \wedge}=V_{1} \wedge V_{2} \wedge \ldots \wedge V_{N}
$$

The Fock space $F$ can be written as

$$
F=\sum_{N=0}^{\infty} \oplus V^{N \wedge}
$$

and any operator $T$ on $F$ can be expressed as the direct sum of the $N$-fold tensor $T^{N}$,

$$
T=\sum_{N=0}^{\infty} \oplus T^{N}
$$


The contraction operator, which defines the reduced density matrix as follows $[1,17,18]$

$$
D^{p}=L_{N}^{p}\left(D^{N}\right),
$$

is a superoperator acting on the $N$ th-order density matrix $D^{N}$, and has following second quantized form (see the Appendix),

$$
L_{N}^{p}\left(T^{N}\right)=\frac{p !}{N !}\left(\Lambda_{-}\right)^{N-p}\left(T^{N}\right),
$$

where $\Lambda_{-}$is defined by

$$
\Lambda_{-}\left(T^{N}\right)=\sum_{i} a_{i}\left(T^{N}\right) a_{i}^{+} .
$$

Equipped with these definitions as well as the Hamiltonian in the occupation number representation,

$$
H=\sum_{i j} h_{i j} a_{i}^{+} a_{j}+\frac{1}{2} \sum_{i j k l} v_{i j k l} a_{i}^{+} a_{j}^{+} a_{l} a_{k},
$$

where $h_{i j}=<i|h| j>$ and $v_{i j k l}=<i j|v| k l>$, we can now deduce the second quantized reduced Bloch equations [19-25]. For the canonical ensemble, the Bloch equation is [1-4]

$$
-\frac{\partial}{\partial \beta} D^{N}=\left(\sum_{i j} h_{i j} a_{i}^{+} a_{j}+\frac{1}{2} \sum_{i j k l} v_{i j k l} a_{i}^{+} a_{j}^{+} a_{l} a_{k}\right) D^{N},
$$

where the $D^{N}$ is regarded as an $N$ th-order irreducible tensor, and $\beta$ is the reverse of the product of the Boltzmann constant $k_{B}$ and absolute temperature $T$. Applying on both sides of above equation with the operator $L_{N}^{p}$ gives

$$
-\frac{\partial}{\partial \beta} D^{p}=\sum_{i j} h_{i j} L_{N}^{p}\left(a_{i}^{+} a_{j} D^{N}\right)+\frac{1}{2} \sum_{i j k l} v_{i j k l} L_{N}^{p}\left(a_{i}^{+} a_{j}^{+} a_{l} a_{k} D^{N}\right) .
$$


The evaluation of the first term of the right hand side of the Eq. (12) results in

$$
\frac{N !}{p !} L_{N}^{p}\left(a_{i}^{+} a_{j} D^{N}\right)=(N-p) \frac{N !}{(p+1) !} a_{j} L_{N}^{p+1}\left(D^{N}\right) a_{i}^{+}+\frac{N !}{p !} a_{i}^{+} a_{j} L_{N}^{p}\left(D^{N}\right),
$$

and the calculation of the second term yields

$$
\begin{aligned}
\frac{N !}{p !} L_{N}^{p}\left(a_{i}^{+} a_{j}^{+} a_{l} a_{k} D^{N}\right)= & \frac{(N-p)(N-p-1) N !}{(p+2) !} a_{l} a_{k} L_{N}^{p+2}\left(D^{N}\right) a_{i}^{+} a_{j}^{+}+ \\
& +\frac{(N-p) N !}{(p+1) !}\left\{a_{j}^{+} a_{l} a_{k} L_{N}^{p+1}\left(D^{N}\right) a_{i}^{+}-a_{i}^{+} a_{l} a_{k} L_{N}^{p+1}\left(D^{N}\right) a_{j}^{+}\right\}+ \\
& +\frac{N !}{p !} a_{i}^{+} a_{j}^{+} a_{l} a_{k} L_{N}^{p}\left(D^{N}\right) .
\end{aligned}
$$

Therefore, the set of reduced Bloch equations for the canonical ensemble in the occupation number representation is

$$
\begin{aligned}
& -\frac{\partial}{\partial \beta} D^{p}=H D^{p}+\frac{N-p}{p+1}\left(\sum_{i j} h_{i j} a_{j} D^{p+1} a_{i}^{+}+\frac{1}{2} \sum_{i j k l} v_{i j k l} a_{j}^{+} a_{l} a_{k} D^{p+1} a_{i}^{+}-\right. \\
& \left.-\frac{1}{2} \sum_{i j k l} v_{i j k l} a_{i}^{+} a_{l} a_{k} D^{p+1} a_{j}^{+}\right)+\frac{(N-p)(N-p-1)}{2(p+2)(p+1)} \sum_{i j k l} v_{i j k l} a_{l} a_{k} D^{p+2} a_{i}^{+} a_{j}^{+} .
\end{aligned}
$$

Define

$$
\hat{v}_{i j k l}=\frac{1}{2}\left(v_{i j k l}-v_{j i k l}\right),
$$

then the above equation can be simplified as

$$
\begin{aligned}
-\frac{\partial}{\partial \beta} D^{p}= & H D^{p}+\frac{N-p}{p+1}\left(\sum_{i j} h_{i j} a_{j} D^{p+1} a_{i}^{+}+\sum_{i j k l} \hat{v}_{i j k l} a_{j}^{+} a_{l} a_{k} D^{p+1} a_{i}^{+}\right)+ \\
& +\frac{(N-p)(N-p-1)}{2(p+2)(p+1)} \sum_{i j k l} \hat{v}_{i j k l} a_{l} a_{k} D^{p+2} a_{i}^{+} a_{j}^{+} .
\end{aligned}
$$

For the grand canonical ensemble, whose $p$ th-order reduced density matrix is defined by [1]

$$
D_{G}^{p}=\sum_{N=p}^{\infty} \oplus\left(\begin{array}{c}
N \\
p
\end{array}\right) L_{N}^{p}\left[D^{N}\right]
$$


similar procedure leads to the following equation

$$
\begin{aligned}
-\frac{\partial}{\partial \beta} D_{G}^{p}= & \bar{H} D_{G}^{p}+\sum_{i j} \bar{h}_{i j} a_{j} D_{G}^{p+1} a_{i}^{+}+\sum_{i j k l} \hat{v}_{i j k l} a_{j}^{+} a_{l} a_{k} D_{G}^{p+1} a_{i}^{+}+ \\
& +\frac{1}{2} \sum_{i j k l} \hat{v}_{i j k l} a_{l} a_{k} D_{G}^{p+2} a_{i}^{+} a_{j}^{+},
\end{aligned}
$$

where

$$
\bar{h}_{i j}=h_{i j}-\mu \delta_{i j},
$$

and

$$
\bar{H}=\sum_{i j} \bar{h}_{i j} a_{i}^{+} a_{j}+\frac{1}{2} \sum_{i j k l} v_{i j k l} a_{i}^{+} a_{j}^{+} a_{l} a_{k} .
$$

This is a set of second quantized reduced Bloch equations for the reduced density matrices in the grand canonical ensemble.

\section{Reduced Density Matrices with Different Orders for Pairing Hamiltonian}

\subsection{The Pairing Hamiltonian and its Eigenvalues and Eigenstates}

Prior to solve the reduced Bloch equations in the case of the canonical ensemble for a pairing Hamiltonian, we need to review its eigenvalue problem as well as related symmetry properties which is discussed in details in paper [26].

Label a set of $2 \lambda$ spin-orbitals $\left|\psi_{i}\right\rangle(\operatorname{dim} V=2 \lambda=r)$ as

$$
1, \ldots, i, \ldots, \lambda, 1^{\prime}, \ldots, i^{\prime}, \ldots, \lambda^{\prime}
$$


where $i^{\prime}=i+\lambda$, and $\mid i^{\prime}>$ represents the pairing of a single-particle state $\mid i>$. The pairing Hamiltonian is defined as [26-29]

$$
\begin{aligned}
H & =\epsilon N-G Q_{+} Q_{-} \\
& =\epsilon \sum_{i=1}^{r} a_{i}^{+} a_{i}-G \sum_{i, j=1}^{\lambda} a_{i}^{+} a_{i^{\prime}}^{+} a_{j^{\prime}} a_{j},
\end{aligned}
$$

where

$$
Q_{+}=\sum_{i=1}^{\lambda} b_{i}^{+}, b_{i}^{+}=a_{i}^{+} a_{i^{\prime}}^{+}
$$

and

$$
Q_{-}=\sum_{i=1}^{\lambda} b_{i}, b_{i}=a_{i^{\prime}} a_{i},
$$

are called the quasi-spin operators. They are the generators of the Lie algebra for the quasi-spin group $S U^{Q}(2)$. The second term of the Hamiltonian indicates that the interaction is only for the pairing states of the particles, and is therefore called the pairing force. The matrix element of the interaction is represented by a constant $G$. When $G>0$, the interaction is repulsive; when $G<0$, the interaction is attractive.

Since the pairing Hamiltonian Eq. (22) exhibits a symplectic symmetry, its $N$-particle eigenstates should be the basis vectors of the irreducible representation space of the symplectic group, and can be characterized by its weight [30]. For the symplectic group $S p(2 \lambda)$ and its corresponding Lie algebra $C_{\lambda}$, the weight is the eigenvalue for the generator,

$$
S_{i i}=a_{i}^{+} a_{i}-a_{i^{\prime}}^{+} a_{i^{\prime}}, i=1,2, \ldots, \lambda
$$

which is the difference of occupation numbers between the pairing spin orbitals $\mid i>$ and $\mid i^{\prime}>$, with the value being 1,0 , or -1 . They can be grouped 
together as a vector

$$
(\vec{S})=\left(s_{1}, s_{2}, \ldots, s_{\lambda}\right)
$$

Furthermore, every irreducible representation can be characterized by the maximal weight. For the symplectic group $S p(2 \lambda)$, this maximal weight takes the form of $\left(1^{\nu} 0^{\lambda-\nu}\right)$, where the number $\nu$ is called the seniority number, and the irreducible representation of the symplectic group can be written as $\left\langle 1^{\nu}>\right.$. However, there is a possibility that the same weight might still correspond to the different basis vectors. To completely distinguish all the basis vectors of an irreducible representation space for the symplectic group, we therefore need to classify the $N$-particle eigenstates according to the following group chain

$$
S p(2 \lambda) \supset S p(2 \lambda-2) \supset \ldots \supset S p(2) .
$$

This means that each basis vector will further be characterized by a group of $\lambda$ seniority numbers:

$$
(\vec{V})=\left(\begin{array}{llll}
\nu_{1} & \nu_{2} & \ldots & \nu_{\lambda}
\end{array}\right)
$$

Thus, the eigenvectors of the pairing Hamiltonian will be completely identified by the particle number $N$, the weight $(\vec{S})$, and seniority number $(\vec{V})$ : $\mid N,(\vec{S}),(\vec{V})>$. They belong to the irreducible representation spaces $<1^{\nu_{1}}>$, $<1^{\nu_{2}}>, \ldots$, and $<1^{\nu_{\lambda}}>$ of the group $S p(2), S p(4), \ldots$, and $S p(2 \lambda)$, respectively, and satisfy the eigenequation

$$
S_{i i}\left|N,(\vec{S}),(\vec{V})>=s_{i}\right| N,(\vec{S}),(\vec{V})>,\left(s_{i}=1,0,-1\right)
$$

The branching rule of $V^{\wedge \Lambda}$ under the group chain (24) of $S_{p}(2 \lambda)$ is

$$
\left[1^{N}\right] \rightarrow<1^{N}>+<1^{N-2}>+\ldots+<1>\text { or }<0>
$$


for $N \leq \lambda$, or

$$
\left[1^{N}\right] \rightarrow<1^{2 \lambda-N}>+<1^{2 \lambda-N-2}>+\ldots+<1>\text { or }<0>
$$

for $N>\lambda$. The dimension of the irreducible space $\left\langle 1^{\nu}>\right.$ is given by

$$
\operatorname{dim}<1^{\nu}>=\left(\begin{array}{c}
2 \lambda \\
\nu
\end{array}\right)-\left(\begin{array}{c}
2 \lambda \\
\nu-2
\end{array}\right) .
$$

The analytical expression for the basis vectors can be constructed as the following form [26]

$$
|N,(\vec{S}),(\vec{V})>=| N,<1^{\nu}>, W>=\sqrt{\frac{(\lambda-\nu-m) !}{m !(\lambda-\nu) !}} Q_{+}^{m} g_{k_{1}}^{+} \ldots g_{k_{y}}^{+} a_{j_{1}}^{+} \ldots a_{j_{x}}^{+} \mid 0>,
$$

where

$$
g_{k}^{+}=\frac{1}{\sqrt{\left(k-\nu_{k}+2\right)\left(k-\nu_{k}+1\right)}}\left[\sum_{i=1}^{k-1} b_{i}^{+}-\left(k-\nu_{k}+1\right) b_{k}^{+}\right],
$$

and

$$
m=\frac{1}{2}(N-\nu) .
$$

In Eq. (28), the $k_{i}$ is the positive number which gives the difference of two consecutive seniority numbers in the $(\vec{V})$ which is two: $\nu_{k}-\nu_{k-1}=2$; the $j_{i}$ means the same thing but its value equals one: $\nu_{|j|}-\nu_{|j|-1}=1$. The sign of $j_{i}$ is decided by the sign of $s_{|j|}:$ if $s_{|j|}=1, j>0$; while if $s_{|j|}=-1, j<0$. In other words, $\pm k_{1}, \ldots, \pm k_{y}$ are the pairing numbers in the corresponding symplectic table $W$, while $j_{1}, \ldots, j_{x}$ are the unpairing numbers appearing in the table, which captures all the features of the basis vectors (28) classified according to the group chain (24) $[26,31,32]$.

The actions of the quasi-spin operators $Q_{+}$or $Q_{-}$on the eigenvectors are

$$
Q_{+}\left|N,(\vec{S}),(\vec{V})>=\frac{1}{2} \sqrt{(N-\nu+2)(r-N-\nu)}\right| N+2,(\vec{S}),(\vec{V})>,
$$


or

$$
Q_{-}\left|N,(\vec{S}),(\vec{V})>=\frac{1}{2} \sqrt{(N-\nu)(r-N-\nu+2)}\right| N-2,(\vec{S}),(\vec{V})>
$$

respectively. Therefore, we can get the analytic expression for the energy eigenvalue as follows

$$
E(\nu)=N \epsilon-\frac{G}{4}(N-\nu)(r-N-\nu+2) .
$$

\subsection{The Exact Solutions of Reduced Bloch Equation}

We are now in a position to solve the reduced Bloch equation (15) for the pairing Hamiltonian (22). For the case of $p=1$, the set of hierarchy equations can be written as

$$
\begin{aligned}
-\frac{\partial}{\partial \beta} D^{1}= & N \epsilon D^{1}-\frac{1}{2}(N-1) G \sum_{i} a_{i^{\prime}}^{+} Q_{-} D^{2} a_{i}^{+}+\frac{1}{2}(N-1) G \sum_{i} a_{i}^{+} Q_{-} D^{2} a_{i^{\prime}}^{+} \\
& -\frac{(N-1)(N-2)}{6} G Q_{-} D^{3} Q_{+} .
\end{aligned}
$$

Since the pairing Hamiltonian is invariant under the symplectic group $S p(2 \lambda)$, the density matrix describing the states of the system can be characterized by its irreducible representations as follows

$$
D^{N}(\beta)=\sum_{\nu} e^{-\beta E(\nu)} D^{N}(\nu)
$$

where

$$
D^{N}(\nu)=\frac{1}{\operatorname{dim}<1^{\nu}>} \sum_{W \in<1^{\nu}>}\left|N,<1^{\nu}>, W><N,<1^{\nu}>, W\right|
$$

and

$$
\operatorname{Tr} D^{N}(\nu)=1 \quad(\nu=N, N-2, \ldots, 1 \text { or } 0)
$$


In addition,

$$
\sum_{\nu} e^{-\beta E(\nu)}=Z(\beta, \nu, N)
$$

For the same reason, the $p$ th-order reduced density matrix of $N$ th-order density matrix can be written as

$$
D^{p}(\beta)=\sum_{\nu, \nu^{\prime}} \omega_{p}\left(\nu, \nu^{\prime}, \beta\right) D^{p}\left(\nu^{\prime}\right)
$$

where

$$
\begin{gathered}
D^{p}\left(\nu^{\prime}\right)=\sum_{W \in<1^{\nu^{\prime}}>}\left|p,<1^{\nu^{\prime}}>, W><p,<1^{\nu^{\prime}}>, W\right| \\
\left(\nu^{\prime}=p, p-2, \ldots, 1 \text { or } 0\right)
\end{gathered}
$$

and

$$
\operatorname{Tr} D^{p}(\beta)=Z(\beta, V, N)
$$

The thermal probability coefficients $\omega_{p}\left(\nu, \nu^{\prime}, \beta\right)$ are to be decided.

According to the branching rule (26) and dimension formula (27), the oneparticle Hilbert space $V$ (denoted as $<1>$ ) is irreducible and its dimension is $r$. Thus

$$
D^{1}(\beta)=\sum_{\nu} \omega_{1}(\nu, 1, \beta) D^{1}(1)
$$

The two-particle Hilbert Space $V^{2 \wedge}$ comprises two irreducible subspaces < $0>$ and $<1^{2}>$ of $S p(2 \lambda)$. Their dimensions are 1 and $\left(\begin{array}{c}r \\ 2\end{array}\right)-1$, respectively. Therefore

$$
D^{2}(\beta)=\sum_{\nu} \omega_{2}(\nu, 0, \beta) D^{2}(0)+\sum_{\nu} \omega_{2}(\nu, 2, \beta) D^{2}(2)
$$

The three-particle Hilbert space $V^{3 \wedge}$ also includes two irreducible subspaces $<1>$ and $<1^{3}>$ of $S p(2 \lambda)$ with dimensions being $r$ and $\left(\begin{array}{c}r \\ 3\end{array}\right)-\left(\begin{array}{c}r \\ 1\end{array}\right)$, 
respectively. Thus

$$
D^{3}(\beta)=\sum_{\nu} \omega_{3}(\nu, 1, \beta) D^{3}(1)+\sum_{\nu} \omega_{3}(\nu, 3, \beta) D^{3}(3) .
$$

The substitution of the expressions (41), (42), and (43) into Eq. (33) then leads to an equation that the above five coefficients satisfy. Together with three trace conditions (40) of $D^{1}, D^{2}$ and $D^{3}$ as well as the $N$-representability conditions among $D^{1}, D^{2}$ and $D^{3}$ as shown below

$$
\begin{aligned}
D^{2}(\beta) & =L_{3}^{2}\left[D^{3}(\beta)\right] \\
& =\sum_{\nu} \omega_{3}(\nu, 1, \beta) L_{3}^{2}\left[D^{3}(1)\right]+\sum_{\nu} \omega_{3}(\nu, 3, \beta) L_{3}^{2}\left[D^{3}(3)\right],
\end{aligned}
$$

and

$$
\begin{aligned}
D^{1}(\beta) & =L_{3}^{1}\left[D^{3}(\beta)\right] \\
& =\sum_{\nu} \omega_{3}(\nu, 1, \beta) L_{3}^{1}\left[D^{3}(1)\right]+\sum_{\nu} \omega_{3}(\nu, 3, \beta) L_{3}^{1}\left[D^{3}(3)\right],
\end{aligned}
$$

it seems that we can completely determine the reduced density matrices for the pairing Hamiltonian. However, due to the complexity of the $N$ representability, it is often replaced by other conditions. Here, we choose the symmetry conditions among $D^{1}, D^{2}$ and $D^{3}$.

Suppose

$$
L_{3}^{2}\left[D^{3}(1)\right]=a_{1} D^{2}(0)+b_{1} D^{2}(2)
$$

From

$$
Q_{+} Q_{-}=\lambda\left(\begin{array}{c}
N \\
2
\end{array}\right) \Gamma_{2}^{N}(|g><g|)
$$

where $\Gamma_{2}^{N}$ is the expansion operator (see references $[17,31]$ or the Appendix), we get

$$
\begin{aligned}
a_{1} & =\operatorname{Tr}\left[|g><g| L_{3}^{2}\left(D^{3}(1)\right)\right] \\
& =\frac{1}{3}(r-2)
\end{aligned}
$$


But

$$
\operatorname{Tr} L_{3}^{2}\left[D^{3}(1)\right]=a_{1}+b_{1} \frac{1}{2}(r+1)(r-2)=r,
$$

we therefore have

$$
b_{1}=\frac{4}{3(r-2)} .
$$

In the same manner, we assume

$$
L_{3}^{2}\left[D^{3}(3)\right]=a_{2} D^{2}(0)+b_{2} D^{2}(2)
$$

and obtain

$$
a_{2}=0, b_{2}=\frac{r(r-4)}{3(r-2)}
$$

The insertion of Eqs. (46) and (51) into Eq. (44) yields

$D^{2}(\beta)=\sum_{\nu} \omega_{3}(\nu, 1, \beta) \frac{r-2}{3} D^{2}(0)+\sum_{\nu}\left[\omega_{3}(\nu, 1, \beta) \frac{4}{3(r-2)}+\omega_{3}(\nu, 3, \beta) \frac{r(r-4)}{3(r-2)}\right] D^{2}(2)$.

Similarly,

$$
\begin{aligned}
L_{3}^{1}\left(D^{3}(1)\right) & =D^{1}(1), \\
L_{3}^{1}\left(D^{3}(3)\right) & =\frac{1}{6}(r+1)(r-4) D^{1}(1),
\end{aligned}
$$

and we get the expression for $D^{1}(\beta)$

$$
D^{1}(\beta)=\sum_{\nu}\left[\omega_{3}(\nu, 1, \beta)+\omega_{3}(\nu, 3, \beta) \frac{1}{6}(r+1)(r-4)\right] D^{1}(1) .
$$

Therefore, with the aid of symmetry conditions, we have expressed the thermal probability coefficients of $D^{1}(\beta)$ and $D^{2}(\beta)$ by those of $D^{3}(\beta)$.

Now we can calculate three terms of the right hand side of Eq. (33) as follows,

$$
\sum_{i} a_{i^{\prime}}^{+} Q_{-} D^{2}(\beta) a_{i}^{+}=\sum_{\nu} \omega_{3}(\nu, 1, \beta) \frac{r-2}{3} \sum_{i=1}^{\lambda}\left|i^{\prime}><i^{\prime}\right|
$$




$$
\begin{aligned}
\sum_{i} a_{i}^{+} Q_{-} D^{2}(\beta) a_{i^{\prime}}^{+} & =-\sum_{\nu} \omega_{3}(\nu, 1, \beta) \frac{r-2}{3} \sum_{i=1}^{\lambda}|i><i|, \\
Q_{-} D^{3}(\beta) Q_{+} & =\sum_{\nu} \omega_{3}(\nu, 1, \beta) \frac{r-2}{2} \sum_{i=1}^{r}|i><i| .
\end{aligned}
$$

Assume

$$
\begin{aligned}
& \omega_{3}(\nu, 1, \beta)=e^{-\beta E(\nu)} \omega_{3}(\nu, 1), \\
& \omega_{3}(\nu, 3, \beta)=e^{-\beta E(\nu)} \omega_{3}(\nu, 3),
\end{aligned}
$$

where

$$
\exp (-\beta E(\nu)), \nu=N, N-2, \ldots, 1,
$$

are linearly independent. Then from the trace condition of $D^{3}(\beta)$,

$\operatorname{Tr} D^{3}(\beta)=\sum_{\nu} \exp (-\beta E(\nu))\left[\omega_{3}(\nu, 1) r+\omega_{3}(\nu, 3) \frac{r}{6}(r+1)(r-4)\right]=\sum_{\nu} e^{-\beta E(\nu)}$,

we get

$$
\omega_{3}(\nu, 1) r+\omega_{3}(\nu, 3) \frac{r}{6}(r+1)(r-4)=1 .
$$

Furthermore, the substitution of Eqs. (56), (57) and (58) into Eq. (33) gives

$$
\sum_{\nu} e^{-\beta E(\nu)}\left[-\frac{G}{4}(N-\nu)(r-N-\nu+2)+\omega_{3}(\nu, 1) G \frac{N(N-1) r(r-2)}{12}\right]=0 .
$$

This leads to

$$
-\frac{G}{4}(N-\nu)(r-N-\nu+2)+\omega_{3}(\nu, 1) G \frac{N(N-1) r(r-2)}{12}=0 .
$$

Hence

$$
\omega_{3}(\nu, 1)=\frac{3(N-\nu)(r-N-\nu+2)}{N(N-1) r(r-2)},
$$

and

$$
\omega_{3}(\nu, 3)=\frac{6}{r(r-4)(r+1)}\left[1-\frac{3(N-\nu)(r-N-\nu+2)}{N(N-1)(r-2)}\right] .
$$


Finally, we obtain the first-order, the second-order, and the third-order reduced density matrices of $N$-particle systems governed by the pairing Hamiltonian at any finite temperature as shown below,

$$
\begin{aligned}
D^{1}(\beta)= & \frac{1}{r} \sum_{i=1}^{r}|i><i| Z(\beta, V, N), \\
D^{2}(\beta)= & \sum_{\nu} e^{-\beta E(\nu)} \frac{(N-\nu)(r-N-\nu+2)}{N(N-1) r} D^{2}(0)+ \\
& +\sum_{\nu} e^{-\beta E(\nu)}\left\{\frac{4(N-\nu)(r-N-\nu+2)}{N(N-1) r(r-2)^{2}}+\frac{2}{(r-2)(r+1)}\right. \\
& \left.\times\left[1-\frac{3(N-\nu)(r-N-\nu+2)}{N(N-1)(r-2)}\right]\right\} D^{2}(2),
\end{aligned}
$$

and

$$
\begin{aligned}
D^{3}(\beta)= & \sum_{\nu} e^{-\beta E(\nu)} \frac{3(N-\nu)(r-N-\nu+2)}{N(N-1) r(r-2)} D^{3}(1)+\sum_{\nu} e^{-\beta E(\nu)} \frac{6}{r(r-4)(r+1)} \\
& \times\left[1-\frac{3(N-\nu)(r-N-\nu+2)}{N(N-1)(r-2)}\right] D^{3}(3) .
\end{aligned}
$$

These density matrices decide the microscopic states of the system, from which we can calculate the thermodynamic properties.

\section{Conclusions}

We have derived the second quantized form of the reduced Bloch equations for both the canonical and grand canonical ensembles. The deduction is based on the occupation number representation of the contraction operator, and is similar to the ones we used before as described in papers [1] and [2]. The equations obtained provide a direct route for exactly solving the reduced density matrices for the systems represented by the model Hamiltonian in 
the occupation number representation. They thereby open an avenue for investigating the microscopic structures of these systems and their relations to the equilibrium thermodynamic properties.

As an example for exactly solving the reduced Bloch equations, we have obtained the reduced density matrices of the pairing Hamiltonian with different orders as shown in Eqs. (66), (67) and (68). One very interesting conclusion we can make is that these reduced density matrices can yield the projection of the ground state of a superconductor in the $N$-particle space $V^{N}$. This is the case when $G<0$ and $N$ is an even number, and the ground state of the paring Hamiltonian (22) is an AEGP (Antisymmetrized Extreme Geminal Power) function $\mid N,\left(0^{\lambda}\right),\left(0^{\lambda}\right)>$. Therefore, if we choose $E(0)$ as the energy zero point and let $\nu=0$ and $\beta \rightarrow \infty$ in Eqs. (66), (67) and (68), we can obtain the reduced density matrices of the ground state for the superconductor as follows

$$
\begin{aligned}
D^{1}(T=0) & =\frac{1}{r} \sum_{i=1}^{r}|i><i|=\frac{1}{r} I_{1}, \\
D^{2}(T=0) & =\frac{r-N+2}{r(N-1)} D^{2}(0)+\frac{2(N-2)}{r(r-2)(N-1)} D^{2}(2) \\
& =\frac{r-N}{(r-2)(N-1)}|g><g|+\frac{2 r(N-2)}{(r-2)(N-1)} D^{1} \wedge D^{1},
\end{aligned}
$$

and

$$
\begin{aligned}
D^{3}(T=0)= & \frac{3(r-N+2)}{(N-1) r(r-2)} D^{3}(1)+\frac{6(N-4)}{r(r-2)(r-4)(N-1)} D^{3}(3) \\
= & \frac{9(r-N)}{r(r-2)(r-4)(N-1)}|g><g| \wedge D^{1}+ \\
& +\frac{6 r^{2}(N-4)}{(r-2)(r-4)(N-1)} D^{1} \wedge D^{1} \wedge D^{1}
\end{aligned}
$$

These results are identical to Coleman's [33]. 
Since there are many other interesting physical systems often modelled in an approximate way by the Hamiltonians with a second quantized form, we expect that the ideas and methodologies we have discussed in this paper can be further explored, and that the fundamental equations (15) or (19) we have derived will find wide applications in the study of their microscopic states as well as their interplay with macroscopic thermodynamic properties.

\section{A Appendix: Second Quantized Form of Con- traction and Expansion Operators}

Consider an antisymmetrized $N$-particle state ket $\mid i_{1} i_{2} \ldots i_{N}>$ composed of

the single-particle states defined in the Section 2. It is easy to see that the action of an annihilation operator $a_{j}$ on this state ket is

$$
a_{j}\left|i_{1} i_{2} \ldots i_{N}>=\sum_{k=1}^{N}(-1)^{N-k} \delta_{j i_{k}}\right| i_{1} i_{2} \ldots \hat{i}_{k} \ldots i_{N}>,
$$

where the superscript ${ }^{\wedge}$ indicates that the state $\mid i_{k}>$ is destroyed. The conjugate of Eq. (70) is

$$
<i_{1} i_{2} \ldots i_{N}\left|a_{j}^{+}=\sum_{k=1}^{N}(-1)^{N-k} \delta_{j i_{k}}<i_{1} i_{2} \ldots \hat{i}_{k} \ldots i_{N}\right| .
$$

The combination of Eqs. (70) and (71) therefore yields

$$
\begin{aligned}
& \sum_{i} a_{i}\left|i_{1} i_{2} \ldots i_{N}><j_{1} j_{2} \ldots j_{N}\right| a_{i}^{+} \\
= & \sum_{k, k^{\prime}}(-1)^{k+k^{\prime}} \delta_{i_{k} j_{k^{\prime}}}\left|i_{1} i_{2} \ldots \hat{i}_{k} \ldots i_{N}><j_{1} j_{2} \ldots \hat{j}_{k^{\prime}} \ldots j_{N}\right| .
\end{aligned}
$$


On the other hand, the state ket $\mid i_{1} i_{2} \ldots i_{N}>$ can be expanded as a determinant as follows,

$$
\left|i_{1} i_{2} \ldots i_{N}>=\frac{1}{\sqrt{N}} \sum_{k=1}^{N}(-1)^{k-1}\right| i_{1} i_{2} \ldots \hat{i}_{k} \ldots i_{N}>\mid i_{k}>_{(N)} .
$$

Thus, the action of contraction operator $L_{N}^{N-1}$ on the basis operator $\mid i_{1} i_{2} \ldots i_{N}><$ $j_{1} j_{2} \ldots j_{N} \mid$ is

$$
\begin{aligned}
& L_{N}^{N-1}\left(\left|i_{1} i_{2} \ldots i_{N}><j_{1} j_{2} \ldots j_{N}\right|\right) \\
= & \frac{1}{N} \sum_{k, k^{\prime}}(-1)^{k+k^{\prime}} \delta_{i_{k} j_{k^{\prime}}}\left|i_{1} i_{2} \ldots \hat{i}_{k} \ldots i_{N}><j_{1} j_{2} \ldots \hat{j}_{k^{\prime}} \ldots j_{N}\right| .
\end{aligned}
$$

The comparison of Eqs. (71) and (74) leads to

$$
L_{N}^{N-1}\left(\left|i_{1} i_{2} \ldots i_{N}><j_{1} j_{2} \ldots j_{N}\right|\right)=\frac{1}{N} \sum_{i} a_{i}\left|i_{1} i_{2} \ldots i_{N}><j_{1} j_{2} \ldots j_{N}\right| a_{i}^{+}
$$

Furthermore, since any $N$ th-order tensor operator $T^{N}$ can be expressed as a linear combination of the basis operators $\left\{\left|i_{1} i_{2} \ldots i_{N}><j_{1} j_{2} \ldots j_{N}\right|\right\}$, we finally get

$$
L_{N}^{N-1}\left(T^{N}\right)=\frac{1}{N} \sum_{i} a_{i} T^{N} a_{i}^{+}
$$

which is the second quantized form for the contraction operator.

The expansion operator $\Gamma_{p}^{N}$ is the reverse operation of the contraction operator $L_{N}^{p}$ and transforms a $p$ th-order tensor to an $N$ th-order one $(p \leq N)$ $[17]$

$$
\Gamma_{p}^{N}\left(T^{p}\right)=A_{N}\left[T^{p} \otimes I^{N-p}\right] A_{N},
$$

where $A_{N}$ is the antisymmetrized operator for $N$ particles. Since the expansion operator $\Gamma_{p}^{N}$ is the adjoint mapping of the contraction operators $L_{N}^{p}$ $[17]$

$$
<\Gamma_{p}^{N}\left(T^{p}\right), T^{N}>=<T^{p}, L_{N}^{p}\left(T^{N}\right)>
$$


we can also obtain its second quantized form as follows

$$
\Gamma_{N-1}^{N}\left(T^{N-1}\right)=\frac{1}{N} \sum_{i} a_{i}^{+} T^{N-1} a_{i}
$$

\section{References}

[1] L. Wei and C. C. Sun, Physica A, in press (2003); e-print, cond-mat/0306306

[2] L. Wei and C. C. Sun, Physica A, in press (2003); e-print, cond-mat/0306307.

[3] F. Bloch, Zeits. f. Physick 74, 295 (1932).

[4] J. G. Kirkwood, Phys. Rev. 44, 31 (1933).

[5] K. Pichler, D. A. Halliday, D. D. C. Bradley, P. L. Burn, R. H. Friend, and A. B. Holmes, J. Phys.: Condens. Matter 5, 7155 (1993).

[6] W. Klemperer, Ann. Rev. Phys. Chem. 46, 1 (1995).

[7] J. Yu, M. Hayashi, S. H. Lin, K.-K. Liang, J. H. Hsu, W. S. Fann, C.-I. Chao, K.-R. Chuang, S.-A. Chen, Synth. Met. 82, 159 (1996).

[8] S. P. Kwasniewski, J. P. Francois, and M. S. Deleuze, J. Phys. Chem. A 107, 5168 (2003). 
[9] H. G. Drickamer and C. W. Franck, Electronic Transitions and the High Pressure Chemistry and Physics of Solids (London, Chapman and Hall, New York, 1973).

[10] W. Kohn and L. J. Sham, Phys. Rev. A 140, 1133 (1965).

[11] (a) B. Lorzao, Nucl. Phys. A397, 225 (1983); (b) B. Lorzao and C. Quesne, Nucl. Phys. A403, 327 (1983).

[12] K. Hagino and G. F. Bertsch, Nucl. Phys. A679, 163 (2000).

[13] J. M. Blatt, Prog. Theor. Phys. 23, 447 (1960).

[14] R. Balian, H. Flocard and M. Veneroni, Phys. Rep. 317, 252 (1999).

[15] B. Weiner and O. Goscinski, Phys. Rev. A 27, 57 (1983).

[16] B. Weiner, H. J. Aa. Jensen and Y. Öhrn, J. Chem. Phys. 80, 2009 (1984).

[17] H. Kummer, J. Math. Phys. 8, 2063 (1967).

[18] J. E. Harriman, Phys. Rev. A 17, 1257 (1978).

[19] L. Cohen and C. Frishberg, Phys. Rev. A 13, 927 (1976).

[20] H. Nakatsuji, Phys. Rev. A 14, 41 (1976).

[21] H. Schlosser, Phys. Rev. A 15, 1349 (1977).

[22] J. E. Harriman, Phys. Rev. A 19, 1893 (1979).

[23] C. Valdemoro, Phys. Rev. A 45, 4462 (1992). 
[24] D. A. Mazziotti, Phys. Rev. A 57, 4219 (1998).

[25] A. J. Coleman, Intern. J. Quantum Chem. 85, 196 (2001).

[26] Z. H. Zeng, C. C. Sun, and A. J. Coleman, in Density Matrices and Density Functionals, pp. 141-165 (1987), eds by R. Erdahl and V. H. Smith Jr. (Dordrecht: Reidel).

[27] S. G. Nilsson and O. Prior, Mat. Fys. Medd. Dan. Vid. Selsk. 32, no 16 (1960).

[28] R. W. Richardson, J. Math. Phys. 18, 1802 (1977).

[29] F. Pan and J. P. Draayer, Phys. Lett. B 442, 7 (1998).

[30] B. G. Wybourne, Classical Groups for Physicists (Wiley, New York, 1974).

[31] Z. H. Zeng and C. C. Sun, J. Mol. Sci. 4, 287 (1986).

[32] A. J. Coleman, J. Math. Phys. 27, 1933 (1986).

[33] A. J. Coleman, J. Math. Phys. 6, 1425 (1965). 